\title{
BRAP-2 promotes DNA damage induced germline apoptosis in $C$. elegans through the regulation of SKN-1 and AKT-1
}

\author{
Dayana R. D'Amora ${ }^{1} \cdot$ Queenie Hu ${ }^{1,2} \cdot$ Monica Pizzardi ${ }^{1}$ Terrance J. Kubiseski $\mathbb{C}^{1,3}$
}

Received: 16 June 2017 / Accepted: 8 November 2017 / Published online: 22 January 2018

(c) ADMC Associazione Differenziamento e Morte Cellulare 2018

\begin{abstract}
As part of the DNA damage response (DDR) network, the tumour suppressor Breast cancer susceptibility gene 1 (BRCA1) is activated to facilitate DNA repair, transcription and cell cycle control. BRC-1, the Caenorhabditis elegans ortholog of BRCA1, has conserved function in DNA double strand break repair, wherein a loss of $b r c-1$ results in high levels of germline apoptosis. BRAP2/IMP was initially identified as a BRCA1 associated binding protein and previously we have shown that the $C$. elegans brap-2 deletion mutant experiences BRC-1 dependent larval arrest when exposed to low concentrations of paraquat. Since BRC-1 function in the germline is conserved, we wanted to determine the role of BRAP-2 in DNA damage induced germline apoptosis in C. elegans. We examined levels of germ cell death following DNA damage and found that brap-2(ok1492) mutants display reduced levels of germline apoptosis when compared to the wild type, and the loss of brap-2 significantly reduced germ cell death in $b r c-1$ mutant animals. We also found increased mRNA levels of skn-1 following DNA damage in brap-2 mutants and that skn-l RNAi knockdown in brap-2;brc-1 double mutants and a loss of pmk-1 mutation in brap-2 mutants increased apoptosis to wild type levels, indicating that brap-2 promotion of cell survival requires PMK-1 and SKN-1. Since mammalian BRAP2 has been shown to bind the AKT phosphatase PHLPP1/2, it suggests that BRAP2 could be involved in the Insulin/Insulin-like growth factor Signaling (IIS) pathway. We found that this interaction is conserved between the C. elegans homologs and that a loss of akt-1 in brap-2 mutants increased germline apoptosis. Thus in response to DNA damage, our findings suggest that BRAP-2 is required to attenuate the pro-cell survival signals of AKT-1 and PMK-1/SKN-1 to promote DNA damage induced germline apoptosis.
\end{abstract}

\section{Introduction}

Activation of apoptosis is an essential process in development and ensures the elimination of abnormal cells that can lead to tumour formation and the onset of cancer [1-3]. In Caenorhabditis elegans, apoptosis is genetically conserved

Edited by E Baehrecke

Electronic supplementary material The online version of this article (https://doi.org/10.1038/s41418-017-0038-7) contains supplementary material, which is available to authorized users.

Terrance J. Kubiseski

tkubises@yorku.ca

1 Department of Biology, York University, Toronto, Ontario, Canada

2 Sunnybrook Research Institute, Toronto, Ontario, Canada

3 Program in Neuroscience, York University, Toronto, Ontario, Canada and functions in the germline to provide cytoplasmic contents and maternal determinants to growing oocytes, as well as remove cells whose DNA has been damaged beyond repair by exogenous stressors such as ionizing radiation (IR), chemicals and toxins [4].

Apoptosis activated by the p53-like protein CEP-1 occurs in the pachytene region of the germline, known as the "death loop" [4]. DNA damage leads to the activation of CEP-1 by upstream DNA damage sensors that phosphorylate and activate CEP-1 [5-7]. CEP-1 transcriptional upregulation of the genes for pro-apoptotic factors EGL-1 and CED-13 release CED-9 inhibition of CED-4 to activate CED-3 for the execution of apoptosis [6]. BRC-1 has been reported to facilitate the conserved repair of DNA double strand breaks (DSBs) using homologous recombination through inter-sister recombination [1]. $b r c-1$ mutants exhibit increased levels of germline apoptosis, increased embryonic lethality and X-chromosome non-disjunction [8].

BRAP2 (BRCA1-binding protein 2 or BRAP as listed in the HUGO database) in mammalian systems has been 
Fig. 1 BRAP-2 promotes DNA damage induced germline apoptosis. a Representative images of germline corpses (indicated with yellow arrows) were detected $24 \mathrm{~h}$ post IR. A significant reduction of apoptosis was observed in brap2 and brap-2;brc- 1 double mutants compared to $\mathrm{N} 2$ and brc-1 mutants. Results represent 3 independent trials. Two trials were completed at $120 \mathrm{~Gy}$. Corpses were scored $24 \mathrm{~h}$ post IR using Acridine Orange (AO). b Following IR, brap-2(ok1492) mutants displayed a significant reduction in apoptosis compared to the wild type (N2). At $60 \mathrm{~Gy}$, brc-1(tm1145) mutants have an incidence of $9.20 \pm 0.90$ corpses, while brap-2;brc-1 double mutants exhibit a 4.5fold decrease in apoptosis with $2.02 \pm 0.17$ corpses. A reduction in apoptosis with a loss of brap2 is also observed at $120 \mathrm{~Gy}$. c Using brap-2 RNAi to knockdown brap-2 expression, following IR a 2.8 -fold reduction in apoptosis was observed in wild type (N2) and brc-1 mutant animals. Worms were grown on brap-2 RNAi bacteria or control pL4440, irradiated at the L4 stage and corpses were scored $24 \mathrm{~h}$ post IR a

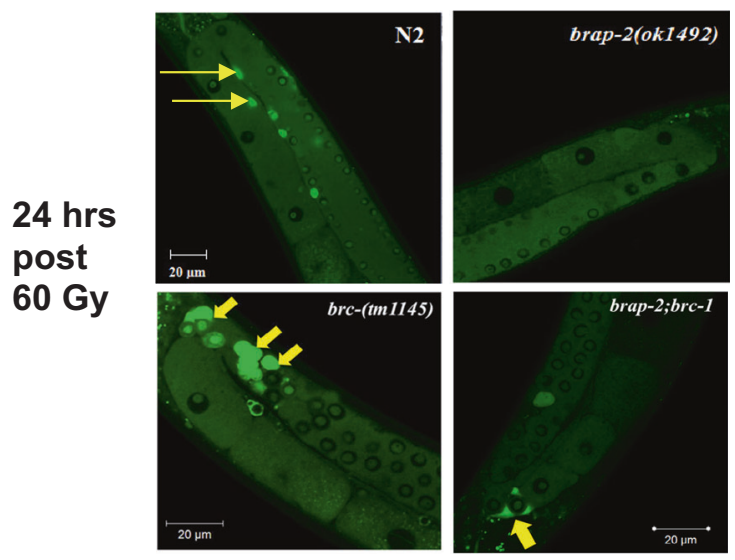

b

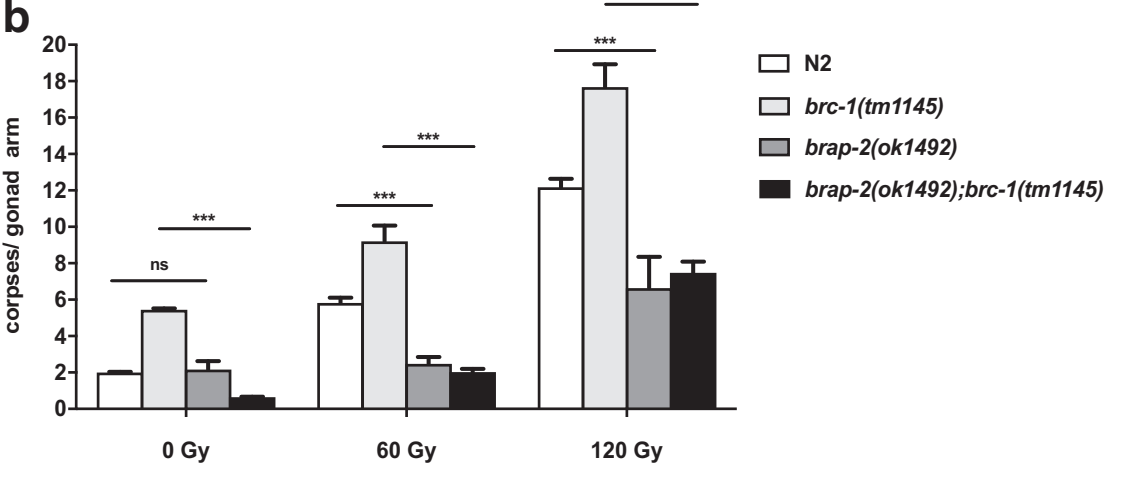

C

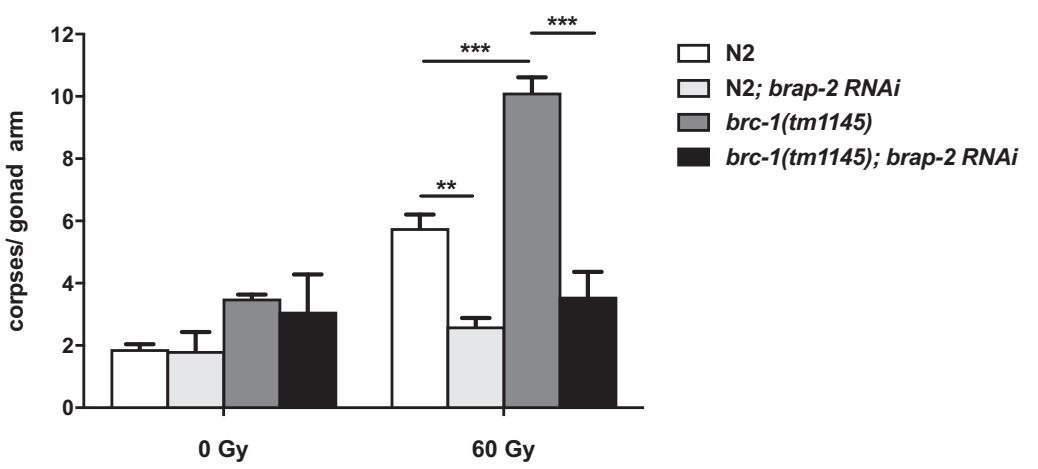

characterized as a RAS-responsive effector protein capable of ubiquitin ligase activity [9]. BRAP2 was first found to bind to the nuclear localization signal (NLS) motifs of BRCA1 and retain BRCA1 in the cytoplasm, preventing its nuclear localization [10]. Recently, BRAP2 has been found to bind to and act as a scaffold protein for PHLPP1, an AKT protein phosphatase, in mouse testes [11]. In C. elegans, BRAP2 is conserved and known as BRAP-2 [2]. When exposed to oxidative stress, brap-2 mutants experience lethality and L1 developmental arrest due to high levels of CKI-1 (p21 ortholog) [2]. Loss of BRC-1 in brap-2 mutants prevented lethality, developmental arrest and decreased CKI-1 levels, establishing a genetic link between BRAP-2 and BRC-1.
Due to the conservation of BRC-1 function in C. elegans and the genetic interaction between BRC-1 and BRAP-2 upon oxidative stress, we examined the role of BRAP-2 in DNA damage induced germline apoptosis. We found that brap-2(ok1492) mutants exhibit a reduction in DNA damage induced germline apoptosis and that germline apoptosis caused by a loss of BRC-1 requires BRAP-2. We determined that brap-2 mutants are dauer defective and the interaction between PHLP-2 (C. elegans homolog of AKT protein phosphatase PHLPP1/2) and BRAP-2 is conserved in C. elegans. We also found that a loss of PMK-1, SKN-1 and AKT-1 in brap-2 mutants increases apoptosis. Taken together, these observations suggest a model where a loss of BRAP-2 limits DNA damage induced germline apoptosis 
and promotes cell survival through regulation of the PMK-1 activated SKN-1/Nrf2 oxidative stress response pathway and the IIS Pathway.

\section{Results}

\section{BRAP-2 is required for DNA damage induced germline apoptosis}

Since BRC-1 functions in the DDR, and having previously established a genetic link between BRC-1 and BRAP-2 during oxidative stress, we wanted to determine whether BRAP-2 is necessary for the induction of germline apoptosis. Unlike $b r c-1$ mutants, we observed a decrease in apoptosis in brap-2(ok1492) mutants compared to the wild type following DNA damage (Fig. 1a). When we introduced the ced-1p::GFP reporter strain into brap-2(ok1492), allowing for both early and late phase apoptotic cells to be detected [12], a 50\% reduction in corpse appearance following DNA damage was observed (Fig. S1). However, physiological levels of apoptosis in brap-2(ok1492) mutants are similar to the wild type (Fig. 1b), indicating that brap-2 may specifically regulate DNA damage induced germline apoptosis.

To test if brap-2 is required for the high levels of germline apoptosis in $b r c-1$ mutants, we generated a brap-2 (ok1492);brc-1(tm1145) double mutant and scored apoptotic levels following IR. We observed a significant reduction in germ cell death at both 60 and 120 Gy (Fig. 1b). When both wild type and brc-1(tm1145) mutants were fed brap-2 RNAi, a similar 2.5-fold reduction in apoptosis was observed (Fig. 1c). These results indicate that excessive apoptosis caused by a loss of brc- 1 is dependent on brap-2. The brap-2(ok1492) mutation does not affect the timing or kinetics of corpse appearance, since $48 \mathrm{~h}$ after DNA damage exposure, brap-2(ok1492) mutants displayed low levels of apoptosis compared to the wild type (Fig. S2).

We determined the expression of BRAP-2 by generating a BRAP-2::GFP strain using CRISPR-Cas9 and found that BRAP-2 is highly expressed in the germline (Fig. S3). To ensure that reduced apoptosis in brap-2 mutants was not caused by a decrease in germ cell number, we scored the number of nuclei before and after DNA damage using whole worm DAPI staining [13] and found that the total number of germline nuclei (nuclei per $\mu^{2}$ ) in brap-2 (ok1492) mutants did not differ significantly compared to the wild type (Fig. S4A). Physiologically, we found that brap-2 mutants experienced a $20 \%$ reduction in total germline nuclei compared to the wild type, which we believe does not account for the consistent $50 \%$ reduction in apoptosis seen in brap-2 mutants following DNA damage (Fig. S4B). In order to determine whether BRAP-2 is a
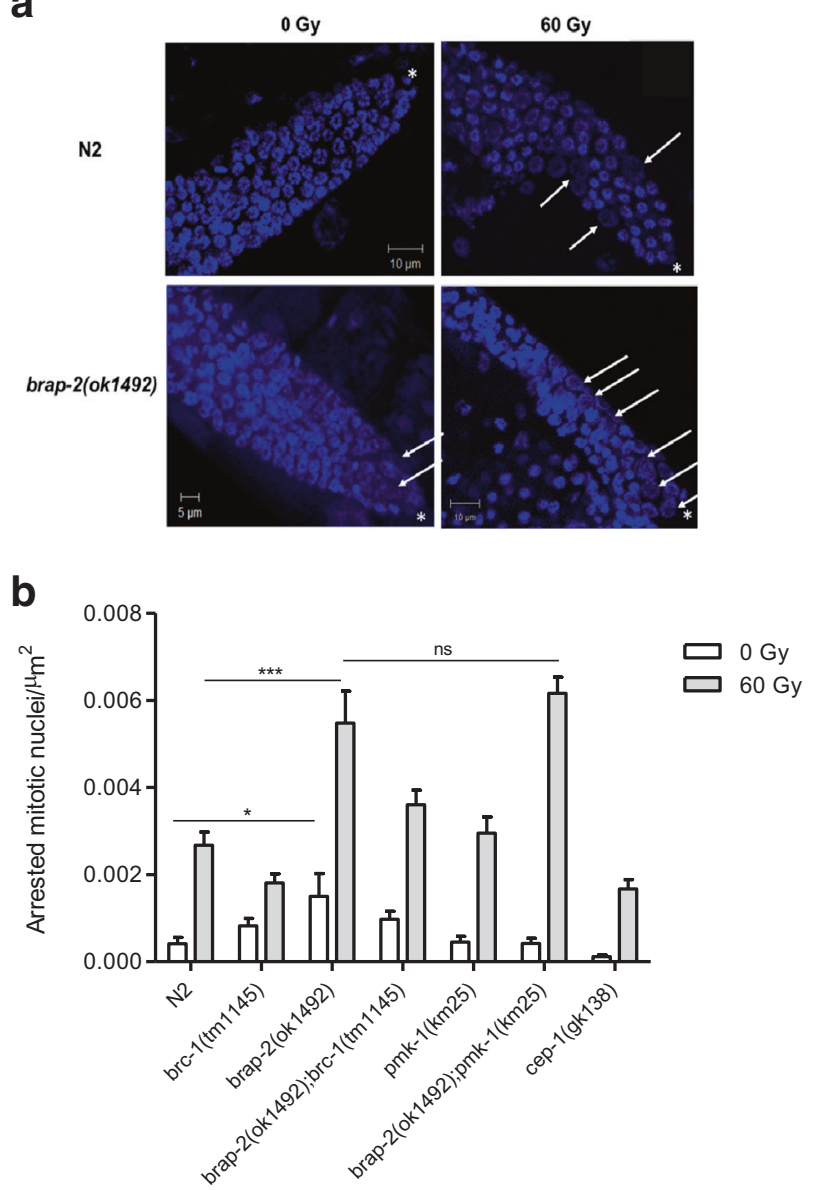

Fig. 2 brap-2(ok1492) mutants exhibit robust mitotic arrest following DNA damage. a Representative confocal images of the mitotic zone (MZ) for each genotype at 0 and $60 \mathrm{~Gy}$. Whole adult worms $(24 \mathrm{~h}$ post L4) were stained with DAPI and images were taken. Arrested nuclei transiently enlarge (indicated by white arrows) and were scored manually using Zen 2010 Software. White asterisk indicates distal tip of the gonad. Results represent $N=16-40$ per genotype. b brap-2 (ok1492) mutants displayed an increase in the number of arrested nuclei per $\mu \mathrm{m}^{2}$ in the MZ compared to the wild type (N2), and is not dependent on PMK-1

involved in the regulation of apoptosis of somatic cells, we scored the number of apoptotic cells in developing embryos directly, using a ced-1p::GFP reporter strain. We found that in 5 distinct stages of embryonic development, brap-2 (ok1492) mutants displayed no significant difference in the number of corpses when compared to the wild type (Fig. S4C).

The 9-1-1 checkpoint (MRT-2, HUS-1, CLK-2) is a complex of protein sensors that interpret DNA damage signals and relay them to induce mitotic arrest in an attempt to repair DNA [5]. If the damage is beyond repair they send signals to ATM-1 and ATL-1 kinases that can activate CEP1 (p53 ortholog) for the induction of apoptosis [5,14]. In the C. elegans germline, nuclei arrested in the mitotic zone can be viewed directly, enlarging following DNA damage to 

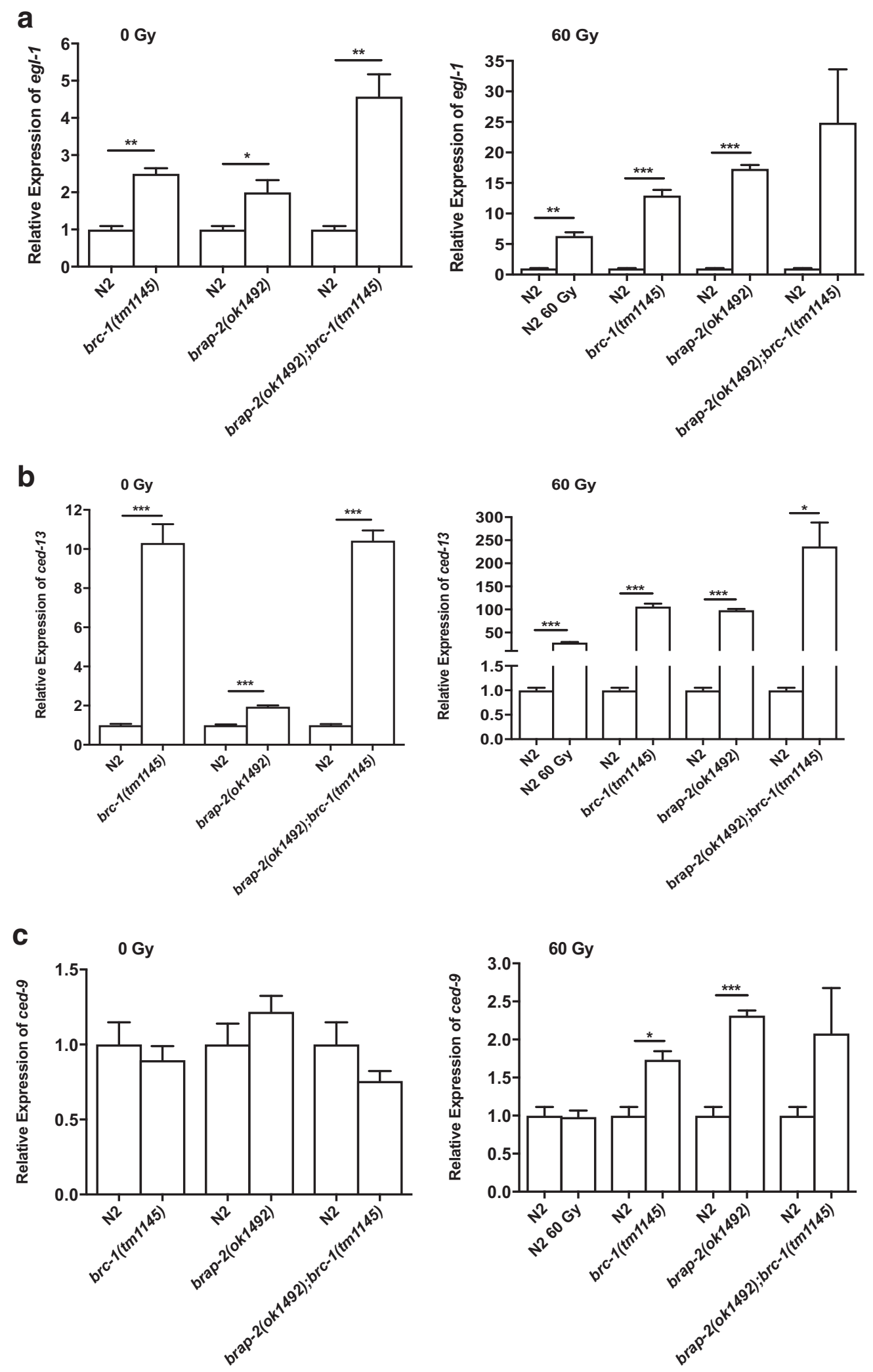

Fig. 3 brap-2(ok1492) increased apoptosis gene expression. Using qRT-PCR, mRNA levels of $\mathbf{a} e g l-1$ and $\mathbf{b}$ ced-13 (pro-apoptotic) were quantified at $0 \mathrm{~Gy}$ and following $60 \mathrm{~Gy}$ of DNA damage. Following IR, levels of egl-1 and ced-13 are enhanced in brap-2(ok1492) mutants. Results represent 3 biological replicates, normalized to act-1

of the wild type (N2) 0 Gy. c Using qRT-PCR, mRNA levels of the anti-apoptotic gene ced-9 were quantified at $0 \mathrm{~Gy}$ and after $60 \mathrm{~Gy}$. Following IR, levels of ced-9 are elevated in brap-2(ok1492) mutants. Results represent 3 biological replicates, normalized to act-1 of $\mathrm{N} 2$ at 0 Gy 
enter G2 phase cell cycle arrest [13]. Interestingly, brap-2 (ok1492) mutants displayed an increase in mitotically arrested nuclei physiologically, and a more robust proportion of arrested nuclei following IR exposure (Fig. 2). Therefore, a loss of BRAP-2 does not (i) alter the timing of corpse appearance, (ii) inhibit germline proliferation, (iii) or prevent mitotic arrest, suggesting that BRAP-2 specifically promotes DNA damage induced germline apoptosis and is required for a $b r c-1$ mutant triggered activation of apoptosis.

DNA damage induced germline apoptosis is activated by a conserved, canonical signaling pathway initiated when CEP-1 up-regulates egl-1 and ced-13 (BH3 orthologs) transcription [15-17]. We asked whether a loss of brap-2 affects CEP-1 function by monitoring expression of egl-1 and ced-13. Surprisingly, brap-2 and brap-2;brc-1 double mutants displayed a significant increase in egl-1 and ced-13 mRNA expression levels both prior to and following DNA damage induction (Fig. 3a, b), indicating that the decrease in apoptosis seen in brap-2(ok1492) mutants is not due to reduced activity of the canonical apoptotic pathway. brap-2 mutants also displayed elevated mRNA levels of ced-9 following DNA damage (Fig. 3c). CED-9 is a "pro-survival" gene that prevents apoptosis and its ablation leads to immense germ cell death and inviability [18]. To investigate the potential interaction between BRAP-2 and CED-9, we generated brap-2;ced-9 double mutants and assessed apoptotic levels and found that the absence of brap-2 in ced-9 mutants failed to reduce the high levels of apoptosis seen in ced-9 mutants (Fig. 4a), indicating that decrease in apoptosis seen in brap-2 mutants probably requires CED-9.

\section{SKN-1 and PMK-1 are required to decrease apoptosis in brap-2;brc-1 mutants}

SKN-1 is essential for the initiation of gene expression against harmful reactive oxygen species (ROS) [19,20]. Previously, we found that brap-2 mutants increased SKN-1 activity, promoting expression of its target phase II detoxification genes [21]. We wanted to determine whether SKN1 is responsible for the brap-2(ok1492) effect on germline apoptosis. While we previously found that $s k n-1$ mRNA levels are increased in brap-2 mutants [21], here we found that brap-2 mutants experience a 2-fold increase in $s k n-1 c$ mRNA levels after IR (Fig S5). We then asked whether the reduction of apoptosis in brap-2 mutants was dependent on SKN-1. Using RNAi, we knocked down skn-1 in brap-2 mutants and scored germline apoptosis before and after IR. We observed that $s k n-1$ RNAi knockdown in wild type worms does not affect apoptosis, as previously reported [22] and that skn-l RNAi knockdown increased apoptosis in brap-2;brc-1 double mutants to levels similar to the wild type (Fig. 4b). pmk-1 RNAi in brap-2;brc-1 double mutants produced a similar increase in levels of apoptosis (Fig. S5C). However, we did not observe increased apoptosis in single brap-2 mutants with $s k n-1$ knockdown. This suggests that a loss of brap-2 promotes cell survival in brc1 mutants, in part, through SKN-1 activation by PMK-1.

Phosphorylation of SKN-1 by PMK-1 (p38 MAPK ortholog) and MPK-1 (ERK1/2 ortholog) promote SKN-1 entry into intestinal nuclei and activation of its target genes $[23,24]$. We asked if PMK-1 and MPK-1 contribute to the reduced apoptosis in brap-2 mutants following DNA damage exposure. We scored apoptotic levels in brap-2; pmk-1 double mutants and found that a loss of pmk-1 in brap-2(ok1492) mutants elevated apoptosis to wild type levels (Fig. 4c). In brap-2(ok1492) mutants, we detected increased endogenous levels of both the nonphosphorylated and phosphorylated forms of ERK1/2 (MPK-1 homolog) (Fig. S6). However, the loss of MPK-1 did not elevate apoptosis in brap-2 mutants (Fig. S7A). In addition, increased mRNA levels of egl-1, ced-13 and ced-9 in brap-2 mutants were not dependent on PMK-1 (Fig. S7B, C). Together, these results suggest that the reduction in apoptosis in brap-2(ok1492) mutants is dependent on PMK1 activity and indicates that reduced levels of apoptosis previously seen in brap-2;brc-1 mutants, may be mediated in part by SKN-1 through PMK-1.

\section{brap-2(ok1492) mutants are dauer defective}

While maintaining our brap-2(ok1492) strain, we observed that brap-2(ok1492) mutants did not appear to enter diapause when starved. Developmental arrest (dauer) is initiated by inactivation of IIS leading to inhibition of AKT-1 and AKT-2 (AKT orthologs), allowing DAF-16 (FOXO ortholog) to enter the nucleus for the transcriptional upregulation of pro-survival and dauer genes [25]. To confirm that brap-2(ok1492) worms fail to enter dauer, we performed a dauer defective assay [26], where following starvation dauer worms were treated with $1 \%$ Sodium Dodecyl Sulfate (SDS). We found that similar to daf-16(mu86) mutants, brap-2(ok1492) mutants were unable to survive SDS treatment (Table 1), indicating that the brap-2(ok1492) mutation may inhibit DAF-16, preventing worms from entering dauer. We also tested the effect of removing MPK1, PMK-1 and AKT-1, and found that they rescued brap-2 mutants and allowed them to enter dauer (Table 1). Interestingly, unlike AKT-1, a loss of AKT-2 in brap-2 mutants did not restore dauer entry. This suggests that BRAP-2 may function specifically through AKT-1 to inhibit DAF-16.

Since brap-2 mutants are dauer defective, we hypothesized that IIS may be enhanced, specifically AKT-1, to decrease DAF-16 activity. To verify whether a loss of brap2 causes increased IIS signaling, we measured the mRNA expression levels of two known targets of this pathway, ins- 
Fig. 4 skn-1 RNAi knockdown increases apoptosis in brap-2;

brc-1 double mutants following DNA damage. a Loss of CED-9 causes massive germline cell death and inviability. A loss of brap-2 in maternally-rescued ced-9 homozygotes produced no significant difference in apoptosis prior to or following DNA damage. Thus a loss of brap-2 does not reduce apoptosis in ced-9 mutants. Worms were irradiated at the L4 stage and corpses were scored using AO $24 \mathrm{~h}$ post IR. Results represent $N=31-41$ and $\mathrm{N}=11-12$ gonads at $0 \mathrm{~Gy}$ and $60 \mathrm{~Gy}$, respectively. b Although skn-1 RNAi in the wild type and brap-2(ok1492) mutants does not affect apoptosis, skn-1 knockdown in brap-2;brc-1 increased apoptosis to levels similar to the wild type (N2). Results represent 3-7 trials. Two trials were completed for $b r c-1$ (tm1145) and brap-2(ok1492) on pL4440 at $0 \mathrm{~Gy}$. Worms were fed control pL4440 or skn-1 RNAi, grown to L4 stage and exposed to 0 and $60 \mathrm{~Gy}$. Apoptotic cells were scored $24 \mathrm{~h}$ post IR using AO. c Following DNA damage, loss of pmk-1 in brap-2 mutants increased apoptosis to levels comparable to N2. Corpses were scored before and after IR using AO staining. Results represent 3 independent trials. For $b r c-1$ and brap-2, at 0 Gy two independent trials each were completed
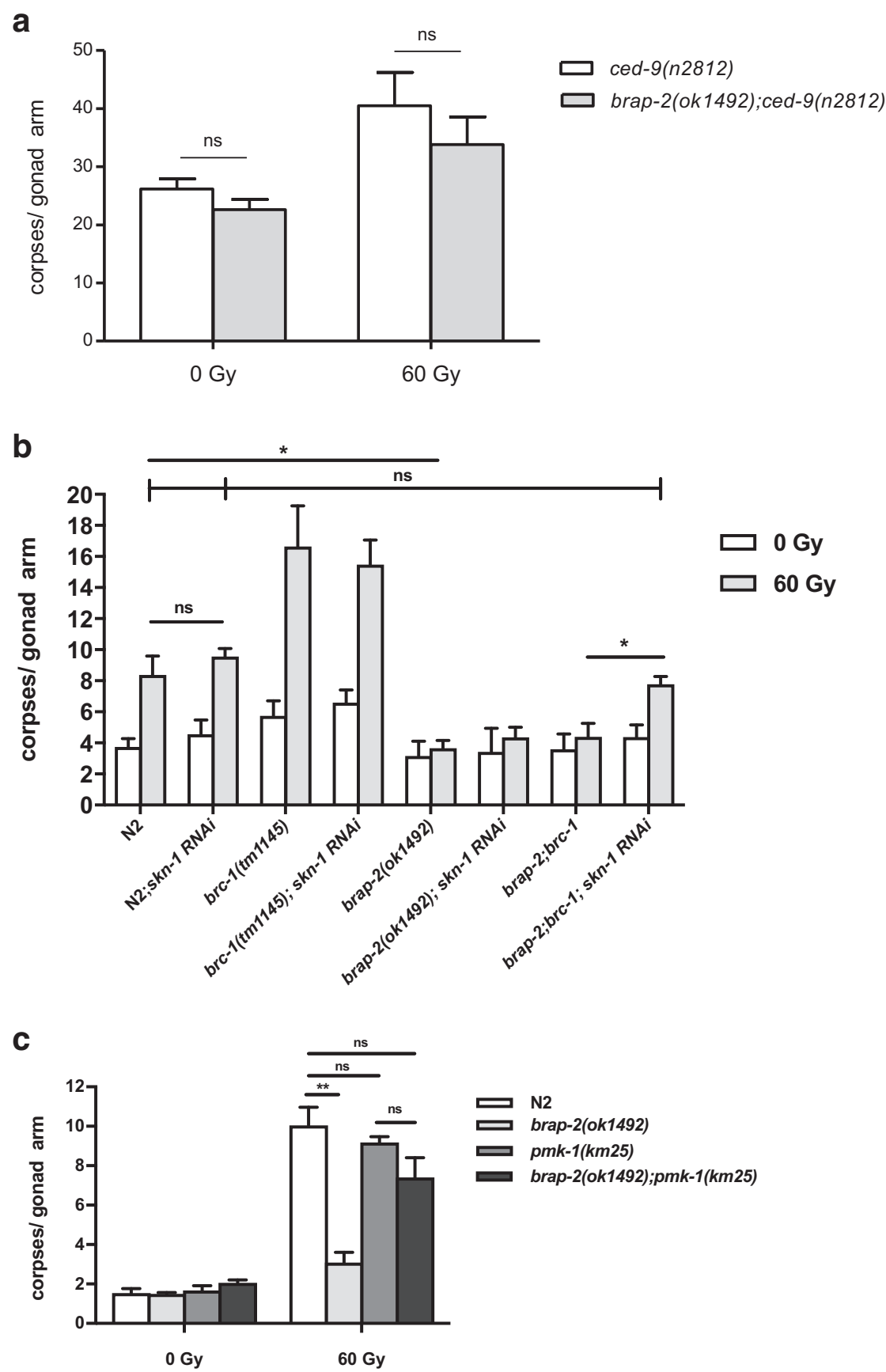

7 and ctl-1 [27,28]. Similar to daf-16 mutants, brap-2 mutants displayed a 2-fold increase in ins-7 expression while ctl- 1 was significantly reduced (Fig. S8).

We also examined the localization of DAF-16 in vivo, using a DAF-16::GFP reporter strain in a brap-2(ok1492) mutant background. As a positive control for DAF-16 nuclear accumulation, worms were treated with $5 \mathrm{mM}$ sodium arsenite. We found that both before and following arsenite treatment, brap-2(ok1492) mutants displayed a reduction in nuclear accumulation of DAF-16 (Fig. S9). We also saw a modest increase in cytoplasmic DAF-16 localization in brap-2 mutants following $60 \mathrm{~Gy}$ of IR. Taken together these results suggest that BRAP-2 regulates IIS, where a loss of BRAP-2 may lead to increased DAF-16 inhibition by AKT-1, both physiologically and when exposed to exogenous stressors.

\section{Loss of akt-1 increased apoptosis in brap-2 mutants}

akt-1 and akt-2 gain of function mutants have been found to suppress DNA damage induced germline apoptosis [29]. Having observed that brap-2 mutants inhibit DAF-16 
Table 1 brap-2 mutants are dauer defective

\begin{tabular}{llll}
\hline Strain & $\begin{array}{l}\text { Number of } \\
\text { plates }(N)\end{array}$ & Thrashing $(\% N)$ & $\begin{array}{l}\text { Dead }(\% \\
N)\end{array}$ \\
\hline $\mathrm{N} 2$ & 70 & 100 & 0 \\
akt-1(ok525) & 46 & 82.6 & 17.4 \\
akt-2(ok393) & 46 & 65.2 & 34.7 \\
daf-16(mu86) & 69 & 0 & 100 \\
brap-2(ok1492) & 61 & 0 & 100 \\
mpk-1(ga111) & 40 & 95 & 5 \\
pmk-1(km25) & 48 & 100 & 0 \\
$\begin{array}{l}\text { akt-1(ok525);brap- } \\
\text { 2(ok1492) }\end{array}$ & 41 & 65.9 & 34.2 \\
$\begin{array}{l}\text { akt-2(ok393);brap- } \\
\text { 2(ok1492) }\end{array}$ & 42 & 4.8 & 95.2 \\
$\begin{array}{l}\text { brap-2(ok1492); } \\
\text { mpk-1(ga111) }\end{array}$ & 44 & 90.9 & 9.1 \\
$\begin{array}{l}\text { brap-2(ok1492); } \\
\text { pmk-1(km25) }\end{array}$ & 37 & 70.3 & 29.7 \\
\hline bap-2(ok1492) & & & \\
\hline
\end{tabular}

brap-2(ok1492) mutants were unable to survive SDS treatment following starvation. Loss of $m p k-1, p m k-1$ and akt-1 but not akt-2 allowed brap-2 mutants to enter dauer and survive SDS treatment. Plates were viewed under a dissection microscope and scored as "Thrashing" if worms were alive and moving in solution. This demonstrates selection for dauers due to their ability to resist SDS. Plates were scored as "Dead" if no worm movement in solution was observed.

function, we hypothesized that BRAP-2 may function through AKT-1 to regulate DNA damage induced germline apoptosis. We generated a akt-1(ok525);brap-2(ok1492) double mutant strain and scored apoptosis following IR. We found that a loss of akt-1 in brap-2 mutants increased apoptosis to wild type levels (Fig. 5a). Interestingly, although a loss of akt-2 in brap-2 mutants increased apoptosis, it was not as significant as a loss of akt-1. Knockdown of akt-1 using RNAi in both brap-2 and brap2; brc-1 double mutants also increased apoptosis to wild type levels following DNA damage (Fig. 5b).

We quantified akt-1 mRNA expression in brap-2 mutants and found that akt-1 levels in brap-2 mutants increased 2.2-fold (Fig. S10A). We also found that the level of endogenous AKT-1 in brap-2 mutants was increased 1.43-fold, while phlp-2 mutants produced no change in AKT-1 (Fig. S10B). We then asked if activation of DAF-16 upon removal of akt- 1 was responsible for the brap-2 mutant reduction in apoptosis; however, the removal of DAF-16 did not increase apoptosis in brap-2 (ok1492) mutants indicating that DAF-16 is not required (Fig. 5a). Together, these results indicate that AKT-1 is at least partially required for the decrease in germline apoptosis seen in brap-2 mutants upon IR, in a daf-16 independent manner.

\section{BRAP-2 interacts with PHLP-2, the C. elegans homolog of the AKT phosphatase PHLPP1/2}

BRAP2 is expressed in many tissues, but has been found most enriched in testes [11]. A yeast 2-hybrid screen using a testes specific cDNA library uncovered several BRAP2 binding partners and one candidate was the AKT protein phosphatase PHLPP1. PHLPP1 dephosphorylates AKT at S473 [30-32] and inactivates it, preventing the inhibition of FOXO and other targets which promote cell death [33]. $C$. elegans contains PHLP-2 (F43C1.1), which is orthologous to PHLPP1 and PHLPP2. We found that BRAP-2 physically binds to PHLP-2 in vitro, indicating that this interaction is conserved (Fig. 6a). Therefore, it is possible that BRAP-2 may function to regulate the activation of the IIS/ AKT pathway in C. elegans.

\section{PHLP-2 is required to promote apoptosis}

Dephosphorylation of AKT by PHLPP1 inactivates AKT, preventing downstream AKT function that leads to cell survival, growth, and proliferation [32]. We hypothesized that loss of PHLP-2 would allow for persistent AKT-1 activation and cause a reduction in germline apoptosis following DNA damage exposure. Physiologically, apoptotic levels in phlp-2(tm7788) mutants was lower than the wild type, and following DNA damage phlp-2 mutants displayed a 4.5-fold reduction in apoptosis (Fig. 6b), indicating PHLP-2 may be required for the general activation of apoptosis. We also found that phlp-2 RNAi knockdown reduced apoptosis in $b r c-1$ mutants (Fig. 6c). Surprisingly, when we generated the akt-1(ok525);phlp-2(tm7788) double mutant strain, we observed a significant reduction in apoptosis following exposure to IR. This indicates PHLP-2 may be required to regulate other proteins besides AKT-1 to promote germline apoptosis (Fig. 6b).

\section{Discussion}

Here we provide evidence of a role for BRAP-2 in the DDR, where it is required to promote DNA damage induced germline apoptosis and the high levels of apoptosis incurred by loss of $b r c-1$. While BRC-1 functions to prevent apoptosis, we found that BRAP-2 promotes apoptosis in the germline upon DNA damage. Previously, we found that brc- 1 was necessary in somatic cells to induce cell cycle arrest in brap-2 mutants upon oxidative stress [2], demonstrating a different response than in the germline. In contrast to somatic cells which suppress apoptosis and the DDR checkpoint, the germline employs the 9-1-1 DNA damage sensor complex and CEP-1 function to activate cell death in 
Fig. 5 Loss of akt-1 increases apoptosis in brap-2 mutants. a Loss of akt-1 significantly increased apoptosis in brap-2 mutants to levels similar to the wild type (N2), following IR. While no change in apoptosis is observed in brap-2 mutants with the loss of daf-16. Results represent 3 independent trials. b akt-1 RNAi in brap-2;brc-1 mutants increased apoptosis to levels similar to N2 following IR. Results represent 3 independent trials. Two independent trials were completed for $b r c-1 ; d a f-16$ mutants a

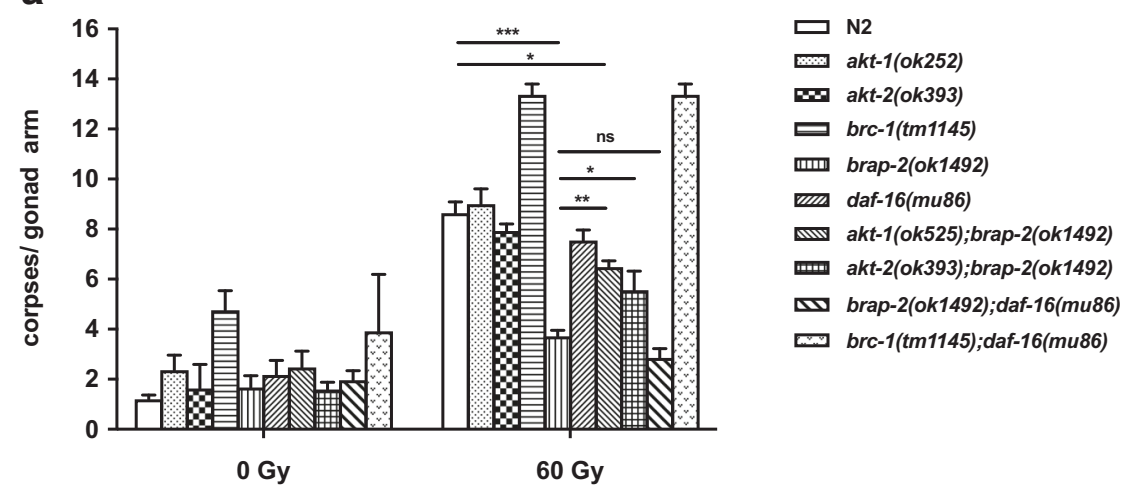

b

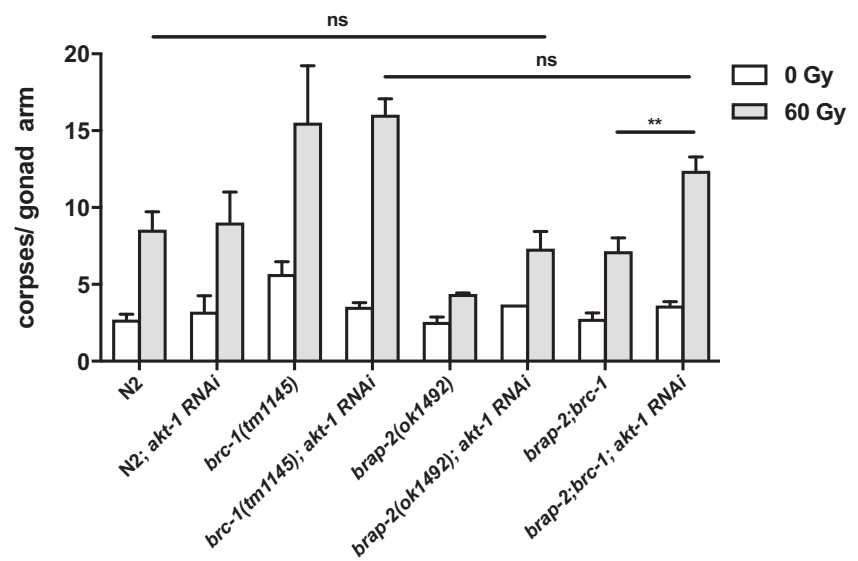

response to DNA damage [34]. Similar to germline cells, somatic cells still express DNA repair genes like BRC-1 to repair DNA damage yet DDR checkpoint signaling is not induced, avoiding apoptosis activation [13,34]. Due to the lack of DDR checkpoint and apoptosis activation in soma compared to the germline, it is possible that the differences in signaling that occur upon DNA damage in these two tissues elicits the activation of diverse downstream effectors to activate distinct responses by BRC-1 and BRAP-2.

Previously, we found that the brap-2(ok1492) mutation increased both SKN-1 localization into intestinal nuclei and expression of phase II detoxification genes in C. elegans [21], revealing BRAP-2 as a negative regulator of SKN-1, whose function is the upregulation of phase II genes for protection against ROS [21]. Although, akt-1 RNAi has been shown to increase SKN-1 nuclear localization [35], our findings indicate that a loss of akt-1 does not affect SKN-1 activity in brap-2 mutants, as increased levels of SKN-1 target genes were not affected by a loss of akt-1 (Fig. S5D,E).

IR can harm cells directly by inducing DSBs and indirectly through "radiolysis", the hydrolysis of water molecules, leading to the production of ROS and the possible disruption of nucleic acids, proteins and lipids [35]. Similar to its mammalian homolog Nrf2 [36], SKN-1's cytoprotective role can be inferred by its maintainenance of cellular homeostasis in response to oxidative stress. While repressed SKN-1 mRNA transcripts have been identified within the germline, SKN-1 protein has only been detected somatically [20,37]. It is possible that SKN-1 has a cell survival role in the germline apart from responding to ROS in the soma similar to Nrf2, which has been shown to possess a pro-survival role in cancer through its upregulation of anti-apoptotic genes, promotion of an unaltered metabolism and changes to ubiquitin-proteasome protein turnover [36]. In C. elegans, SKN-1 activity may enhance cell survival in the germline through a "soma to germline interaction", a relationship characterized previously in the cell non-autonomous regulation of apoptosis [38], lipid regulation [39], germline proliferation [40] and lifespan extension [41].

AKT-1 and AKT-2 dimerize to phosphorylate and inhibit DAF-16, preventing dauer entry [42]. AKT-1 and AKT-2 function has been found to be uncoupled in germline apoptosis following DNA damage [42]. AKT-1 was found to negatively regulate $\mathrm{CEP}-1$ transcriptional activity and the activation of apoptosis [29] independent of DAF-16, while DAF-2 was found to function downstream or independently of AKT-1, regulating apoptosis through AKT-2 and DAF16 [42]. Similarly, we observed separated AKT isoform 
Fig. 6 Interaction between PHLPP1 and BRAP2 in $C$. elegans is conserved. a Full length BRAP-2 interacts with PHLP-2 in vitro, suggesting that this interaction is conserved in C. elegans. Plasmids were cotransfected in 293 T HEK cells, followed by a GST pull down and western blot analysis. Result represents 3 independent trials. b Loss of phlp-2 reduced germline apoptosis in akt-1 mutants. phlp2 mutants displayed a significant reduction in apoptosis before and after IR. Loss of phlp-2 significantly reduced apoptosis in akt-1 mutants following IR. c phlp-2 RNAi caused a modest reduction in apoptosis in $\mathrm{N} 2$ and brc-1 mutants. Two independent trials were completed for pL4440 and 3 independent trials for phlp-2 RNAi a $\begin{array}{llllll}\text { BRAP-2::GST } & - & - & - & + & + \\ \text { PHLP-2::3XFLAG } & - & + & + & - & + \\ \text { GST } & + & + & - & - & -\end{array}$

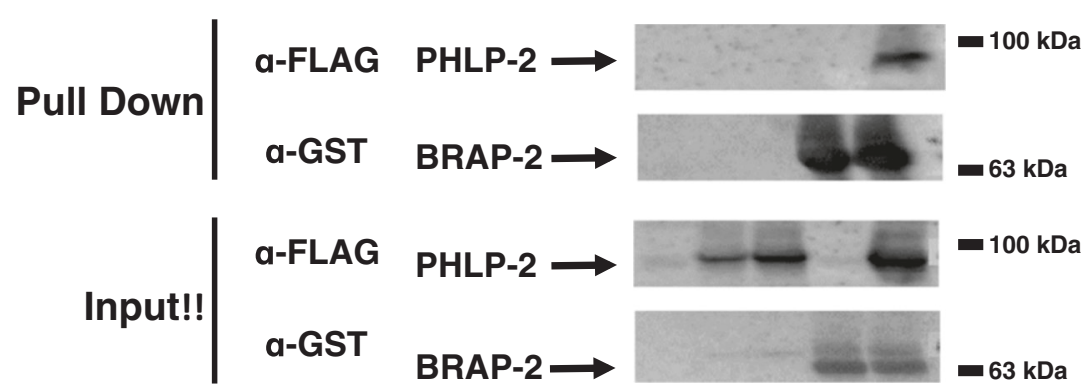

b
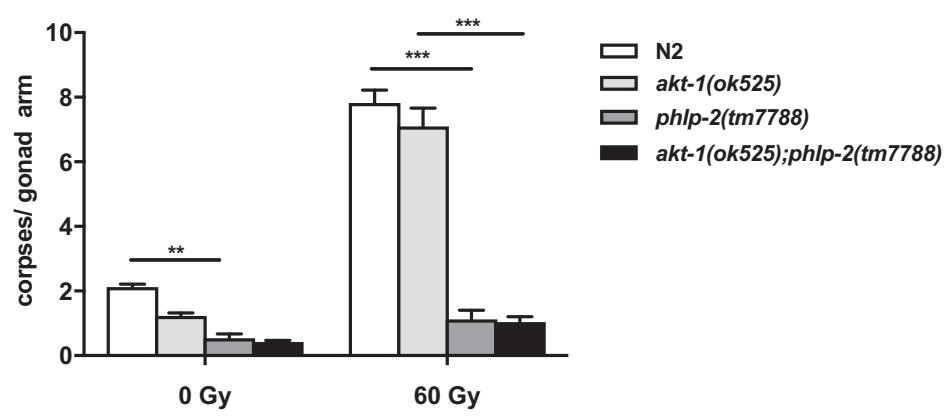

C

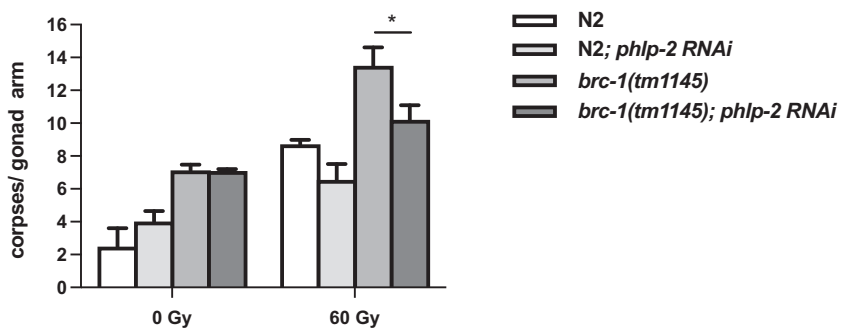

function in brap-2 mutants, where a loss of akt-1 restored dauer and increased germline apoptosis more significantly in brap-2 mutants than a loss of akt-2, in a daf-16 independent manner. Like DAF-2, this indicates that BRAP-2 function may be independent of the components upstream of AKT-1.

Although we were unable to test phosphorylated AKT-1 levels to verify whether this increase in AKT-1 corresponded to increased AKT-1 activation, we found that a loss of brap-2 enhanced IIS stimulation and DAF-16 attenuation. We also confirmed that BRAP-2 interacts with PHLP-2 in vitro, placing BRAP-2 as a potential regulator of the IIS pathway. Therefore, we postulate that AKT-1 and SKN-1 activity are modulated by BRAP-2 for regulation of DNA damage induced germline apoptosis. We also identified a potential novel role for PHLP-2 in germline apoptosis where $p h l p-2$ mutants displayed a significant and general reduction in germline apoptosis that was not restored with a loss of PMK-1 (Fig. S10C). Like its mammalian ortholog,
BRAP-2 possesses the functional domains of an E3 ubiquitin ligase [2]. We did not verify whether this enzymatic activity is conserved however, it cannot be ruled out as a possible mechanism to post translationally modify and regulate proteins such as PHLP-2, and therefore affect the downstream signaling of these pathways. Similar to brap-2, further investigation is required to determine whether phlp2 directly influences the core apoptotic pathway or functions through an alternate mechanism to regulate germ cell death.

MPK-1 is required for germline progression and activation of CEP-1 [43], while PMK-1 has been linked to germline apoptosis induced by IR, arsenite and bacterial infection [44]. We have found that BRAP-2 may regulate CEP-1 and the core apoptotic pathway through MPK-1, while influencing apoptosis through PMK-1. Our findings suggest that BRAP-2 regulates DNA damage induced germline apoptosis by blocking the activation of pro-cell survival pathways to promote apoptosis by inhibiting 


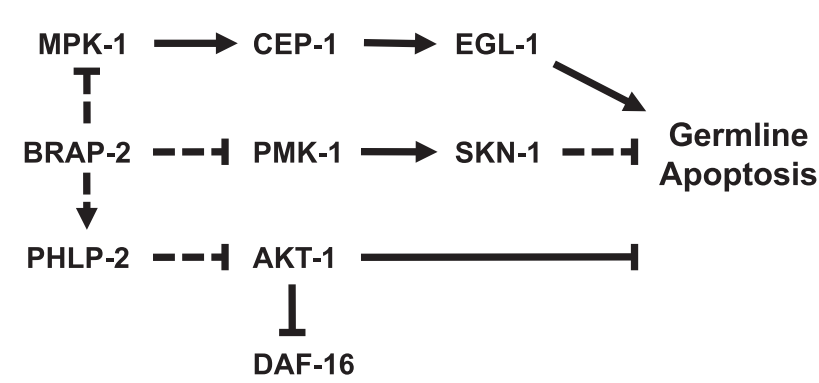

Fig. 7 Proposed model for BRAP-2 regulation of IR/DNA damage induced germline apoptosis. Our findings suggest that BRAP-2 is required for IR/DNA damage induced germline apoptosis by inhibiting SKN-1 (through PMK-1/p38 MAPK) and AKT-1 (through PHLP-2). While loss of BRAP-2 leads to activation of MPK-1, PMK-1 and AKT-1, the pro-survival signals of SKN-1 and AKT-1 inhibit apoptosis despite the activation of the CEP-1/EGL-1 apoptotic pathway. Arrows represent activation, bar headed lines represent inhibition, solid lines represent previously established pathways, while dashed lines indicate interactions proposed from this study

SKN-1 and AKT-1 through PMK-1 and PHLP-2 respectively (Fig. 7). Although further investigation is required, we reveal a potential novel role for PHLP-2, where it may be necessary for the general induction of germline apoptosis.

In addition to mutations in BRCA1, RAS and PI3K/AKT pathway dysfunction has been detected in breast cancer, leading to metastasis and reduced apoptosis [42]. In mammalian systems, it is predicted that BRAP2 prevents BRCA1 from traveling to the nucleus and performing functions that protect genomic integrity [10]. Although, to our knowledge, an apoptotic role for BRAP2 has not be identified, we predict that in combination with mutations in BRCA1, BRAP2 inactivation could promote tumorigenesis, by avoiding death and enhancing cell survival.

Apoptosis is vital for shaping organs and limbs, eliminating self-reactive cells and for protection against malignant transformation. The evasion of apoptosis is a hallmark of cancer and this study has provided us with a genetic tool to better understand the complex and intricate regulation of signaling pathways that govern the balance between pro-death and pro-cell survival factors that lead to apoptosis activation, while discovering potential new targets that regulate apoptosis for future study and therapeutic development.

\section{Materials and methods}

\section{C. elegans strains and genetics}

All worm strains were cultured under standard conditions, as previously described [43]. Strains were obtained from the Caenorhabditis Genetics Center at the University of Minnesota and from the National Bioresource Project in
Tokyo, Japan. Double mutant strains were generated according to standard protocols. The N2 Bristol strain was used as the wild type, and unless noted otherwise all experiments were conducted at $20^{\circ} \mathrm{C}$. C. elegans strains used in this study are listed in Supplemental Material (Table S1).

\section{Fluorescence microscopy}

Worms were picked and anesthetized using $2 \mathrm{mM}$ Levamisole (Sigma-Aldrich, Oakville, ON, Canada) and mounted on $2 \%$ agarose pad. Images of fluorescent worms were taken using a Zeiss LSM 700 confocal laser-scanning microscope with Zen 2010 Software.

\section{Worm synchronization}

For all worm assays worms were synchronized via Egg Lay, as described by the Morimoto Lab (http://groups.molbiosci. northwestern.edu/morimoto/research/Protocols).

\section{Irradiation}

Worms were synchronized to the L4 stage and exposed to ionizing radiation using a ${ }^{137} \mathrm{Cs}$ source (in coordination with the Derry Lab, McMaster Building \& Sick Kids Peter Gilgan Research Tower) at indicated doses. Worms were allowed to recover for $18-24 \mathrm{~h}$ on NGM plates before apoptosis, RNA extraction and qRT-PCR, cytological, and cell cycle arrest analyses.

\section{Germline apoptosis assay}

Worms were allowed to recover for 18-24 $\mathrm{h}$ and corpses (apoptotic cells) were scored using Acridine Orange (Sigma-Aldrich) staining, as previously described [44,45]. After hatching, $m p k-1$ (gall1) worms were grown at $26^{\circ} \mathrm{C}$ (restrictive temperature) [46-48]. Worms were anesthetized using $2 \mathrm{mM}$ Levamisole in M9 buffer and mounted on $2 \%$ agarose pads where 15-20 gonad arms per genotype were scored per treatment. Apoptotic cells in developing embryos were scored in specific embryonic stages directly using the ced-1p::GFP strain.

\section{DNA staining}

To quantify germline nuclei, whole worms were stained with DAPI, as previously described [13]. Z-stack images were taken and using Zen 2010 Lite software the free-form surface area measurement tool was used to measure gonad surface area of one representative slice. Germlines were dissected and stained with DAPI (Vector Labs, Burlingame, CA, USA). ImageJ (National Institute of Health) Cell 
Counter plugin was used to determine the total number of nuclei per zone in one gonad arm per worm with 40-50 worms per genotype imaged and counted.

\section{Generation of BRAP::GFP transgenic strain}

The transgenic strain BRAP-2::GFP was generated, as described previously [49]. The $5^{\prime} \mathrm{HR}$ arm was amplified using a fosmid template and sub-cloned into NaeI/SalI site of pDD282 (Addgene) using the HiFi Assembly Kit (NEB, Whitby, ON, Canada). 3'HR arm was amplified using the fosmid template and was then sub-cloned into pDD282 in SpeI and AvrII site to create the final plasmid. The single guide RNA (sgRNA) was used to mutate the pDD162 (Addgene) vector.

\section{Whole worm staining}

Antibody staining was performed as previously described by the Koelle Lab protocol [50]. Synchronized L4 worms were collected, stained with the designated antibodies and were mounted on $3 \mu \mathrm{L}$ Prolong Gold Anti-fade reagent (Fisher, Waltham, MA USA). Worms were then visualized with Zeiss LSM 700 confocal microscope at 20x. Fluorescence intensity was quantified from 20 worms for each genotype using ImageJ software. Antibodies used were: anti-ERK1/2(1:500) (Santa Cruz, Dallas, TX, USA), anti-pERK1/2 (1:500) (SigmaAldrich), and DAPI $(1 \mu \mathrm{g} / \mathrm{mL})$ (Sigma-Aldrich). Alexa Fluor 488 (1:1000) (Invitrogen) secondary antibody was used for visualization.

\section{RNA interference analysis}

Worms were synchronized on RNA interference (RNAi) plates (NGM containing $0.4 \mathrm{mM}$ IPTG, $100 \mu \mathrm{g} / \mathrm{mL}$ Ampicillin and $12.5 \mu \mathrm{g} / \mathrm{mL}$ Tetracycline) and fed with E. coli HT115 (DE3), transcribing double stranded RNA (dsRNA) from fusion bacterial plasmids homologous to the target with pL4440 used as the control. When animals reached the 1st day of adulthood they were then treated and assayed for germline corpse quantification. The $s k n-1$ RNAi plasmid was obtained from a transcription factor specific RNAi library, as described [51]. The akt-1 RNAi plasmid was purchased from GE Dharmacon (Lafayette, CO, USA). The brap-2 RNAi construct was made by amplifying region 1-843 nucleotides of the brap-2 ORF by PCR, using the EST yk1134a01 as template, and cloning into the KpnI site of pL4440. The phlp-2 RNAi construct was made by amplifying the 26182800 nucleotide region of the predicted phlp-2 ORF (F43C1.1; Wormbase Version WS258) by PCR, using the EST yk1149d03 as template, and cloning into the KpnI site of pL4440.
RNA isolation and quantitative real time PCR

Worm RNA Isolation, quantitative real time PCR (qRTPCR) and data analysis was performed, as described previously [21]. mRNA expression levels for each strain was compared relative to the wild type (N2) control, where the housekeeping gene act-1 was used as the endogenous control for normalization. Data represents three biological replicates. To verify equal primer amplification efficiency, a standard curve for each set of primers was constructed using a serial dilution of cDNA. All primers used in this study are listed in Table S3.

\section{DAF-16 localization assay}

DAF-16 expression was viewed in vivo using cell type specific reporter strain CF1139. To investigate DAF-16 expression in a brap-2(ok1492) mutant background, transgenic strains were generated using standard $C$. elegans crossing technique and confirmed by Single Worm PCR. Worms were synchronized to L4 stage and were treated with $5 \mathrm{mM}$ sodium arsenite (Sigma-Aldrich) in M9 Buffer for $1.5 \mathrm{~h}$ rotating to induce acute stress [52]. Worms were then paralyzed in $2 \mathrm{mM}$ Levamisole in M9 buffer and mounted on $2 \%$ agarose pads. For each genotype, worms were visualized and DAF-16::GFP expression was scored as cytoplasmic, intermediate or nuclear [53].

\section{Dauer defective assay}

The dauer defective assay was performed, as previously described [26]. Singleadult worms from each strain were picked onto individual OP50 seeded NGM plates and allowed to grow for 2 weeks to enter dauer developmental arrest. Each plate was flooded with $1 \mathrm{~mL}$ of $1 \%$ SDS solution and allowed to sit for $30 \mathrm{~min}$. Each plate was then observed under a stereomicroscope to assess the state of the worms. If worms were found to be thrashing on the plate they were declared to have survived (proper entry into dauer). If worms were found to be motionless they were deemed dead (failed to enter dauer). For each genotype, a minimum of 40 starved plates were examined.

\section{Plasmid construction}

To construct 3xFLAG::PHLP-2, the C-terminal region of PHLP-2 (1428 bp) was amplified from worm cDNA and the product was cloned in frame to a BamHI and EcoRI digested pCMV 7.1 (3xFLAG) vector. The N-terminal region of PHLP-2 (1682 bp) was amplified from synthesized DNA (BioMatik, Cambridge, ON, Canada) based on Wormbase sequence for F43C1.1 into a NotI and BglII 
digested pCMV 7.1 (3xFLAG) vector containing the PHLP2 C-terminal region using the HI-FI Assembly Mix (NEB) following the manufacturer's protocol.

\section{GST pull down and western blot analysis}

Human Embryonic Kidney (HEK) 293 T cells were cultured in Dulbecco's Modified Eagle's Medium supplemented with $10 \%$ Fetal Bovine Serum. To test for interaction between GST::BRAP-2 and 3xFLAG::PHLP-2, $5 \mu \mathrm{g}$ DNA of each construct coding these proteins were cotransfected for $48 \mathrm{~h}$ using polyethylenimine (SigmaAldrich) and Optimem (Fisher). Cells were lysed in lysis buffer (1X TBS, $1 \%$ NP-40, $1 \%$ Glycerol, $150 \mathrm{mM} \mathrm{NaCl}$, and complete protease inhibitor cocktail (Sigma-Aldrich) and Glutathione Sepharose Beads (GE Healthcare Life Sciences, Mississauga, ON, Canada) were added according to the manufacturer's protocol with one modification: washing of beads was carried out $6 \mathrm{x}$ for $15 \mathrm{~min}$. Cell and worm lysate samples were analyzed by standard SDS polyacrylamide gel, followed by Western Blot, as previously described [21]. The following antibodies were used: anti-FLAG M2 (Sigma-Aldrich), anti-ERK1/2 (Santa Cruz), anti-pERK1/2 antibody (Sigma-Aldrich) anti-GST (NEB), anti-tubulin (NEB), anti-AKT-1 528, and anti-AKT-1 127. Proteins were detected using SuperSignal West Femto Maximum Sensitivity Substrate (ThermoScientific) and blots were imaged using the DNR Bio Imaging Systems Microchemi System. Band density was measured and normalized to their respective anti-tubulin protein levels using the gel analyzer plugin in ImageJ software.

\section{Statistical analysis}

Statistical analysis was performed with GraphPad Prism® 7 Software. Statistical significance was determined using Two-way ANOVA and Bonferroni post tests for comparison between different groups. Student's $t$-test was performed when comparing two means. A chi-square test was used for analysis of DAF-16::GFP localization [54]. $P$ values of $<0.05$ were taken to indicate statistical significance $\left(* * * p<0.001,{ }^{* *} p<0.01, * p<0.05\right)$. Error bars represent \pm standard error of the mean.

Acknowledgements A number of strains were provided by the CGC, which is funded by NIH Office of Research Infrastructure Programs (P40 OD010440) and the National BioResource Project. AKT antibodies were a kind gift from W.B. Derry of Hospital for Sick Children, Toronto. We thank the Derry Lab (E. Chapman, B. Yu, B. Lant, A. Mateo, M. Hall, M. Gunda and M. Haeri) for their assistance with the irradiation. D.R.D. is a recipient of the Ontario Graduate Scholarship. M.P. is a recipient of the Dean's Award from the Faculty of Science,
York University and a Natural Sciences and Engineering Research Council of Canada (NSERC) undergraduate studentship. D.R.D., Q. H., M.P. and T.J.K were supported by a grant from NSERC.

\section{Compliance with ethical standards}

Conflict of interest The authors declare that they have no conflict of interest.

\section{References}

1. Adamo A, Montemauri P, Silva N, Ward JD, Boulton SJ, La Volpe A. BRC-1 acts in the inter-sister pathway of meiotic double-strand break repair. EMBO Rep. 2008;9:287-92.

2. Koon JC, Kubiseski TJ. Developmental arrest of Caenorhabditis elegans BRAP-2 mutant exposed to oxidative stress is dependent on BRC-1. J Biol Chem. 2010;285:13437-43.

3. Fuchs Y, Steller H. Programmed cell death in animal development and disease. Cell. 2011;147:742-58.

4. Gartner A, Boag, Peter, Blackwell TK. Germline survival and apoptosis. WormBook. 2008;1-20.

5. Jolliffe AK, Derry WB. The TP53 signaling network in mammals and worms. Brief Funct Genom. 2013;12:129-41.

6. Ahmed S, Alpi A, Hengartner MO, Gartner A. C. elegans RAD-5/ CLK-2 defines a new DNA damage checkpoint protein. Curr Biol. 2001;11:1934-44.

7. Harris J, Lowden M, Clejan I, Tzoneva M, Thomas JH, Hodgkin $\mathrm{J}$, et al. Mutator phenotype of Caenorhabditis elegans DNA damage checkpoint mutants. Genetics. 2006;174:601-16.

8. Boulton SJ, Martin JS, Polanowska J, Hill DE, Gartner A, Vidal M. BRCA1/BARD1 orthologs required for DNA repair in Caenorhabditis elegans. Curr Biol. 2004;14:33-9.

9. Chen C, Lewis RE, White MA. IMP modulates KSR1dependent multivalent complex formation to specify ERK1/2 pathway activation and response thresholds. J Biol Chem. 2008;283:12789-96.

10. Li S, Ku C-Y, Farmer AA, Cong Y-S, Chen C-F, Lee W-H. Identification of a novel cytoplasmic protein that specifically binds to nuclear localization signal motifs. J Biol Chem. 1998;273:6183-89.

11. Fatima S, Wagstaff KM, Loveland KL, Jans DA. Interactome of the negative regulator of nuclear import BRCA1-binding protein 2. Sci Rep. 2015;5:9459.

12. Lant B, Derry WB. Fluorescent visualization of germline apoptosis in living Caenorhabditis elegans. Cold Spring Harb Protoc. 2014;2014:420-7.

13. Craig AL, Moser SC, Bailly AP, Gartner A. Methods for studying the DNA damage response in the Caenorhabdatis elegans germ line. Methods Cell Biol. 2012;107:321-52.

14. Bailly A, Gartner A. Germ cell apoptosis and DNA damage responses. Adv Exp Med Biol. 2013;757:249-76.

15. Conradt B, Horvitz HR. The C. elegans protein EGL-1 is required for programmed cell death and interacts with the Bcl-2-like protein CED-9. Cell. 1998;93:519-29.

16. Schumacher B, Schertel C, Wittenburg N, Tuck S, Mitani S, Gartner A, et al. C. elegans ced-13 can promote apoptosis and is induced in response to DNA damage. Cell Death Differ. 2005;12:153-61.

17. Salinas LS, Maldonado E, Navarro RE. Stress-induced germ cell apoptosis by a p53 independent pathway in Caenorhabditis elegans. Cell Death Differ. 2006;13:2129-39. 
18. Malin JZ, Shaham S. Cell death in C. elegans development. Curr Top Dev Biol. 2015;114:1-42.

19. Park S-K, Tedesco PM, Johnson TE. Oxidative stress and longevity in Caenorhabditis elegans as mediated by SKN-1. Aging Cell. 2009;8:258-69.

20. An JH, Blackwell TK. SKN-1 links C. elegans mesendodermal specification to a conserved oxidative stress response. Genes Dev. 2003;17:1882-93.

21. Hu Q, D'Amora DR, MacNeil LT, Walhout AJM, Kubiseski TJ. The oxidative stress response in Caenorhabditis elegans requires the GATA transcription factor ELT-3 and SKN-1/Nrf2. Genetics. 2017;206:1909-22.

22. Ross AJ, Li M, Yu B, Gao MX, Derry WB. The EEL-1 ubiquitin ligase promotes DNA damage-induced germ cell apoptosis in $C$. elegans. Cell Death Differ. 2011;18:1140-9.

23. Inoue $\mathrm{H}$, Hisamoto $\mathrm{N}$, An JH, Oliveira RP, Nishida E, Blackwell $\mathrm{TK}$, et al. The C. elegans p38 MAPK pathway regulates nuclear localization of the transcription factor SKN-1 in oxidative stress response. Genes Dev. 2005;19:2278-83.

24. Kell A, Ventura N, Kahn N, Johnson TE. Activation of SKN-1 by novel kinases in Caenorhabditis elegans. Free Radic Biol Med. 2007;43:1560-6.

25. Hesp K, Smant G, Kammenga JE. Caenorhabditis elegans DAF16/FOXO transcription factor and its mammalian homologs associate with age-related disease. Exp Gerontol. 2015;72:1-7.

26. Malone EA, Inoue T, Thomas JH. Genetic analysis of the roles of daf-28 and age- 1 in regulating Caenorhabditis elegans dauer formation. Genetics. 1996;143:1193-205.

27. Zheng S, Liao S, Zou Y, Qu Z, Liu F. ins-7 gene expression is partially regulated by the DAF-16/IIS signaling pathway in Caenorhabditis elegans under Celecoxib intervention. PLoS ONE. 2014;9:e100320.

28. Lu L, Zhao X, Zhang J, Li M, Qi Y, Zhou L. Calycosin promotes lifespan in Caenorhabditis elegans through insulin signaling pathway via daf-16, age-1 and daf-2. J Biosci Bioeng. 2017;124:1-7.

29. Quevedo C, Kaplan DR, Derry WB. AKT-1 regulates DNAdamage-induced germline apoptosis in C. elegans. Curr Biol. 2007;17:286-92.

30. Newton AC, Trotman LC. Turning off AKT: PHLPP as a drug target. Annu Rev Pharmacol Toxicol. 2014;54:537-58.

31. Gao T, Furnari F, Newton AC. PHLPP: a phosphatase that directly dephosphorylates Akt, promotes apoptosis, and suppresses tumor growth. Mol Cell. 2005;18:13-24.

32. Grzechnik AT, Newton AC. PHLPPing through history: a decade in the life of PHLPP phosphatases. Biochem Soc Trans. 2016;44:1675-82.

33. Narasimhan SD, Mukhopadhyay A, Tissenbaum HA. InAKTivation of insulin/IGF-1 signaling by dephosphorylation. Cell Cycle Georget. Tex 2009;8:3878-84.

34. Vermezovic J, Stergiou L, Hengartner MO, d'Adda, di Fagagna F. Differential regulation of DNA damage response activation between somatic and germline cells in Caenorhabditis elegans. Cell Death Differ. 2012;19:1847-55.

35. Tullet JMA, Hertweck M, An JH, Baker J, Hwang JY, Liu S, et al. Direct inhibition of the longevity-promoting factor SKN1 by insulin-like signaling in C. elegans. Cell. 2008;132: 1025-38.

36. Geismann C, Arlt A, Sebens S, Schäfer H. Cytoprotection "gone astray": Nrf2 and its role in cancer. OncoTargets Ther. 2014;7:1497-518.

37. Page BD, Diede SJ, Tenlen JR, Ferguson EL. EEL-1, a Hect E3 ubiquitin ligase, controls asymmetry and persistence of the SKN-1 transcription factor in the early C. elegans embryo. Dev Camb Engl. 2007;134:2303-14.

38. Ito S, Greiss S, Gartner A, Derry WB. Cell-nonautonomous regulation of C. elegans germ cell death by kri-1. Curr Biol. 2010;20:333-8.

39. Steinbaugh MJ, Narasimhan SD, Robida-Stubbs S, Moronetti Mazzeo LE, Dreyfuss JM, Hourihan JM et al. Lipid-mediated regulation of SKN-1/Nrf in response to germ cell absence. eLife. 2015;4:1-30.

40. Michaelson D, Korta DZ, Capua Y, Hubbard EJA. Insulin signaling promotes germline proliferation in C. elegans. Dev Camb Engl. 2010;137:671-80.

41. McCormick M, Chen K, Ramaswamy P, Kenyon C. New genes that extend $C$. elegans' lifespan in response to reproductive signals. Aging Cell. 2012;11:192-202.

42. Perrin AJ, Gunda M, Yu B, Yen K, Ito S, Forster S, et al. Noncanonical control of C. elegans germline apoptosis by the insulin/ IGF-1 and Ras/MAPK signaling pathways. Cell Death Differ. 2013;20:97-107.

43. Brenner S. The genetics of Caenorhabditis elegans. Genetics. 1974;77:71-94.

44. Kelly KO, Dernburg AF, Stanfield GM, Villeneuve AM. Caenorhabditis elegans msh-5 is required for both normal and radiation-induced meiotic crossing over but not for completion of meiosis. Genetics. 2000;156:617-30.

45. Lant B, Derry WB. Methods for detection and analysis of apoptosis signaling in the C. elegans germline. Methods San Diego Calif. 2013;61:174-82.

46. Leacock SW, Reinke V. Expression profiling of MAP kinasemediated meiotic progression in Caenorhabditis elegans. PLoS Genet. 2006;2:e174.

47. Church DL, Guan KL, Lambie EJ. Three genes of the MAP kinase cascade, mek-2, mpk-1/sur-1 and let-60 ras, are required for meiotic cell cycle progression in Caenorhabditis elegans. Dev Camb Engl. 1995;121:2525-35.

48. Eberhard R, Stergiou L, Hofmann ER, Hofmann J, Haenni S, Teo $\mathrm{Y}$, et al. Ribosome synthesis and MAPK activity modulate ionizing radiation-induced germ cell apoptosis in Caenorhabditis elegans. PLoS Genet. 2013;9:e1003943 http://www.ncbi.nlm.nih. gov/pmc/articles/PMC3836707/

49. Dickinson DJ, Pani AM, Heppert JK, Higgins CD, Goldstein B. Streamlined genome engineering with a self-excising drug selection cassette. Genetics 2015;200:1035-49.

50. Michael Koelle. Antibody Staining of C. elegans [Internet]. In: Michael Finney (ed.). Koelle Lab, Yale School of Medicine; 1998. https://medicine.yale.edu/lab/koelle/protocols/Antibody\%20Sta ining_180540_21947.pdf

51. MacNeil LT, Pons C, Arda HE, Giese GE, Myers CL, Walhout AJM. Transcription factor activity mapping of a tissue-specific in vivo gene regulatory network. Cell Syst. 2015;1:152-62.

52. Wang S, Teng X, Wang Y, Yu H-Q, Luo X, Xu A, et al. Molecular control of arsenite-induced apoptosis in Caenorhabditis elegans: roles of insulin-like growth factor-1 signaling pathway. Chemosphere. 2014;112:248-55.

53. Schafer JC, Winkelbauer ME, Williams CL, Haycraft CJ, Desmond RA, Yoder BK. IFTA-2 is a conserved cilia protein involved in pathways regulating longevity and dauer formation in Caenorhabditis elegans. J Cell Sci. 2006;119:4088-100.

54. Wu C-W, Wang Y, Choe KP. F-Box Protein XREP-4 is a new regulator of the oxidative stress response in Caenorhabditis elegans. Genetics. 2017;206:859-71. 